\title{
Acquired immunoparalysis in paediatric intensive care: prospective observational study
}

\author{
Mark Peters, Andy Petros, Garth Dixon, David Inwald, Nigel Klein
}

The increase in high technology medicine, complex surgical techniques, and use of immunosuppressive agents means that more patients are managed with broad spectrum antibiotics. Such regimens may contribute to the emergence of multidrug resistant micro-organisms, ${ }^{1}$ and many authorities are now rationalising use of antibiotics in hospital to try to limit the spread of antibiotic resistant organisms. For this strategy to be successful, however, it should be combined with attempts to limit the requirement for antibiotic treatment in patients in hospital. ${ }^{1}$

Recent studies indicate that many inpatients may be predisposed to infectious complications because of altered host defences. A subgroup of adults in intensive care with severe sepsis has been shown to express low amounts of HLA-DR on circulating monocytes; this was associated with a relatively immunodeficient state termed monocyte deactivation ${ }^{2}$ or immunoparalysis. These patients improved after being given the cytokine interferon gamma. These observations suggest that focusing on host defences may provide an additional strategy for reducing antimicrobial resistance in hospitals.

\section{Subjects, methods, and results}

We undertook a prospective study of admissions to the paediatric intensive care unit to determine whether monocyte deactivation also occurred in critically ill children. Monocyte HLA-DR expression was determined by flow cytometric analysis of $50 \mu \mathrm{l}$ of blood after staining with fluorochrome labelled monoclonal antibodies against HLA-DR and CD14. A minimum of 2000 monocyte events was collected and the results expressed as median fluorescence intensity.

We studied five groups: 23 consecutive admissions to the paediatric intensive care unit for single system failure (isolated head injury, bronchiolitis due to infection with respiratory syncitial virus, and viral laryngotracheobronchitis); 18 consecutive admissions for severe sepsis and multiple organ system failure ${ }^{3}$ with three or more systems failing, nine patients being studied further in follow up outpatient visits 14-28 days after admission; 11 children undergoing routine outpatient investigations; and five healthy adult laboratory staff. Analysis was performed within 18 hours of admission and repeated roughly 48 hourly for patients with multiorgan system failure.

Monocyte HLA-DR expression was similar among the healthy adults (mean 45.7 (95\% confidence interval 41.8 to 49.6$)$ ), children undergoing routine outpatient investigation (44.4 (35.0 to 53.8)), and children reviewed after recovery from multiple organ system failure (50.5 (40.2 to 60.7)). However, children with multiple organ system failure (17.5 (13.5 to 21.4)) and those with single system failure (26.9 (18.5 to 35.2$)$ ) had significantly reduced HLA-DR expression $(\mathrm{P}<0.05$, one way analysis of variance with least

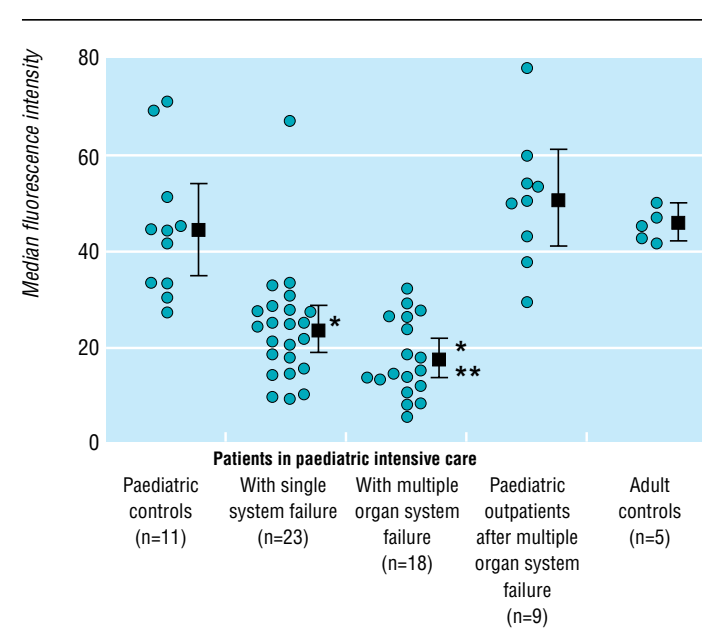

Fig 1 Median fluorescence intensity of monocyte HLA-DR expression in control paediatric outpatients, cases in paediatric intensive care unit because of single or multiple organ system failure, paediatric patients receiving outpatient follow up after multiple system failure, and healthy adults. Individual values and means with $95 \%$ confidence intervals (bars) are shown for each group ( ${ }^{\star} P<0.05$, analysis of variance $v$ control groups). HLA-DR expression was also significantly lower in children with multiple organ system failure than those with single system failure $\left({ }^{\star \star} P<0.05\right)$

significant difference after correction for multiple comparisons) (figure). HLA-DR expression was significantly lower among children with multiple organ system failure than among those with single system failure $(\mathrm{P}=0.045$, two sample $t$ test). Expression remained low throughout their stay in the paediatric intensive care unit (data not shown).

\section{Comment}

This study shows that all children requiring intensive care may be at increased risk of nosocomial infections because of acquired immunoparalysis. Monocyte HLA-DR expression fell in children with single or multiple organ system failure, although more so in the more severely ill children. Adults show similar falls in HLA-DR expression after systemic insults (neurosurgery, burns, trauma, and sepsis). ${ }^{25}$ Previous studies have indicated that patients with monocyte deactivation are at an increased risk of infectious complications. ${ }^{45}$ When those studies are taken together with ours severely ill patients of all ages seem to be predisposed to infections as a result of these alterations in host defences.

Future strategies to decrease nosocomial infection and the emergence of multiresistant micro-organisms should address the capacity of the host to resist infection in hospital environments.

Funding: MP was supported by the Dunhill Medical Trust, GD by the Royal College of Physicians, and DI by SIMS-Portex.

Competing interests: None declared.
Portex Department of Anaesthesia, Intensive Therapy and Respiratory Medicine, Institute of Child Health, London

WC1N 3EH

Mark Peters lecturer in paediatric intensive care

Paediatric Intensive Care Unit, Great Ormond Street Hospital for Children NHS Trust, London WC1N 3JH

Andy Petros

consultant intensivist

Immunobiology

Unit, Institute of

Child Health

Garth Dixon

research fellow

David Inwald

research fellow

Nigel Klein

senior lecturer

Correspondence to: Dr Peters m.peters@ich. ucl.ac.uk

BMJ 1999;319:609-10 
Contributors: AP had the original idea for this study, which was then designed and planned by him and MP and NK. Initial assessment and optimisation of laboratory methods was undertaken by GD, DI, and MP, who subsequently collected and analysed the patient data. The paper was written by MP, NK, and $\mathrm{AP}$ with contributions from GD and DI. MP and NK are guarantors of this study.

1 Wise R, Hart T, Cars O, Streulens M, Helmuth R, Huovinen P, et al. Antimicrobial resistance. $B M J$ 1998;317:609-10.
2 Docke WD, Randow F, Syrbe U, Krausch D, Asadullah K, Reinke P, et al. Monocyte deactivation in septic patients: restoration by IFN-gamm treatment. Nat Med 1997;3:678-81.

3 Wilkinson JD, Pollack MM, Ruttimann UE, Glass NL, Yeh TS. Outcome of pediatric patients with multiple organ system failure. Crit Care Med 1986;14:271-4.

4 Asadullah K, Woiciechowsky C, Docke WD, Liebenthal C, Wauer H, Kox $\mathrm{W}$, et al. Immunodepression following neurosurgical procedures. Crit Care Med 1995;23:1976-83.

5 Schinkel C, Sendtner R, Zimmer S, Faist E. Functional analysis of monocyte subsets in surgical sepsis. J Trauma 1998;44:743-8.

(Accepted 5 March 1999

\title{
Ethnic differences in use of hormone replacement therapy: community based survey
}

\author{
Tess J Harris, Derek G Cook, Paul D Wicks, Francesco P Cappuccio
}

Department of General Practice and Primary Care, St George's

Hospital Medica School, London SW17 0RE

Tess J Harris lecturer

Department of Public Health

Sciences,

St George's

Hospital Medical

School

Derek G Cook

professor

Paul D Wicks

statistician

Blood Pressure Unit, Department of

Medicine,

St George's

Hospital Medical

School

Francesco P

Cappuccio

reader

Correspondence to: Professor Cook

d.cook@sghms.ac.uk

BMJ 1999;319:610-1
Hormone replacement therapy is widely promoted to prevent cardiovascular disease and osteoporosis and relieve menopausal symptoms, although concern exists that much of the cardiovascular effect may be due to its selection by healthy women. ${ }^{1}$ Little is known about its use by women from different ethnic groups in the United Kingdom, particularly women of south Asian origin, who are at increased risk of coronary heart disease, ${ }^{2}$ osteoporosis, ${ }^{3}$ and diabetes ${ }^{4}$ compared with white women.

\section{Subjects, methods, and results}

A population based survey was carried out in Wandsworth, south London, where roughly 12\% of residents are from the Caribbean or west Africa (that is, of African descent) and 6\% are of Indian, Pakistani, or Bangladeshi origin (that is, south Asian). Women aged 40-59 were invited from nine general practices as part of a cardiovascular screening study. ${ }^{4}$ The response rate was $66 \%(941 / 1429)$. Of the 941 women screened, 882 were from one of the ethnic groups being studied.

Ethnic group was recorded at interview on the basis of answers to several questions, including questions on country of birth, language, religion, history of migration, and parental country of birth. Women were asked to bring someone to translate if they could not speak English. This analysis is restricted to data recorded at the interview, as we were interested in factors in the history that could have influenced hormone prescribing. Proportions were compared with $\chi^{2}$ and Fisher's exact tests. The odds of current hormone use was modelled using logistic regression.

White ethnic group, hysterectomy, smoking, and greater age were associated with current hormone use and were included in the model to produce adjusted odds ratios (table). All except smoking remained independently associated with hormone use. Adjusting for practice made little difference to the estimated odds ratios for other variables (data not shown). Restricting analyses to women over 50 gave similar differences between ethnic groups.

Among white women those who had had a hysterectomy were twice as likely to be hormone users as those who had not $(54 \%(7 / 13)$ v 23\% (63/269), $\mathrm{P}=0.02)$. The difference was similar among women of
African descent $(32 \%(6 / 19)$ v 14\% (45/314), $\mathrm{P}=0.05)$ but was not seen among south Asian women (8\% $(1 / 12) v 10 \%(24 / 238), \mathrm{P}=1.0)$. Differences in the effect of hysterectomy between ethnic groups were not significant $(\mathrm{P}=0.44)$.

A greater proportion of women from the Caribbean than from Africa used hormone replacement $(19 \%(40 / 214)$ v $9 \%(10 / 107))$. Similarly, women of Pakistani origin were more likely to be users $(17 \%$ $(6 / 36))$ than women of Indian $(10 \%(18 / 185))$ or Bangladeshi origin $(3 \%(1 / 29))$. None of these differences, however, was significant at the $5 \%$ level. All of these rates were lower than the $25 \%$ found in white women.

\section{Comment}

Women from ethnic minority groups were less likely than white women to use hormone replacement therapy. The differences were independent of other factors, including smoking, age, and hysterectomy. As others have reported, ${ }^{5}$ women with cardiovascular disease were not more likely to be prescribed hormone replacement, although it is often advised. This suggests continuing uncertainty about risks and benefits.

Our study has potential limitations, including response bias. However, those who refused to take part were comparable to the population surveyed for all aspects considered. ${ }^{4}$ The prevalence of current hormone use in white women that we found is similar to other recent work in the United Kingdom, ${ }^{5}$ lending validity to our findings.

The differences in use of hormone replacement reported here have not to our knowledge been described before in the United Kingdom. They are important, because possible long term protective effects on heart and bone may particularly benefit south Asian women. Uptake of other preventive health measures is lower in south Asian than white or African-Caribbean women in the United Kingdom.

Hormone replacement assessment provides an opportunity for health promotion, assessing cardiovascular risk factors, and discussing cervical and breast screening. Opportunities for these discussions with women from ethnic minority groups may be being missed. Further research is needed, particularly among 